\title{
Phase Transition of Two-Dimensional Chiral Supramolecular Nanostructure Tuned by Electrochemical Potential
}

\author{
Gui-jin Su,*,t,‡ Zhi-hai Li, ${ }^{\ddagger}$ and R. Aguilar-Sanchez ${ }^{\ddagger, \S}$
}

\begin{abstract}
State Key Laboratory of Environmental Chemistry and Ecotoxicology, Research Center for Eco-Environmental Sciences, Chinese Academy of Sciences, P.O. Box 2871, Beijing 100085, China, Institute of Bio-and Nanosystems 3, Research Center Jülich, D-52425 Jülich, Germany, and Facultad de Ciencias Quimicas, Universidad Autonoma de Puebla, 72570 Puebla, Mexico
\end{abstract}

Molecular chiralty and phase transition of $p$-phenylenedi $(\alpha-$ cyanoacrylicacid) di-n-ethyl ester ( $p$-CPAEt) assembled on $\mathrm{Au}(111)$ have been studied in the electric double layer region in $0.1 \mathrm{M} \mathrm{HClO}_{4}$ by electrochemical scanning tunneling microscopy (ECSTM) technique. Three types of chiral supramolecular nanostructures were resolved at differently charged interfaces. Within a potential range $(0.65 \mathrm{~V}<E<0.8 \mathrm{~V}$, region I), a close-packed physisorbed adlayer of chiral stripe pattern, with the $(3 \times 6)$ structure, has been observed. At more negative potential $(0.2 \mathrm{~V}<E \leq 0.65 \mathrm{~V}$, region II), the stripe patterns gradually dissolved, and two types of new chiral network structures $(3 \sqrt{ } 7 \times 4 \sqrt{ } 7)$ and $(3 \sqrt{ } 7 \times$ $3 \sqrt{ } 7$ ) evolved on reconstructed and unreconstructed surfaces, respectively. On the basis of the high-resolution STM images, it was tentatively proposed that three types of chiral supramolecular nanostructures were formed by two-dimensional adorption-induced chiral p-CPAEt species together with lateral hydrogen-bonding interaction $(\mathrm{C}-\mathrm{H} \cdots \mathrm{N} \equiv \mathrm{C})$. Intriguingly, ECSTM images allow in situ monitoring of the phase transition process of these chiral adlayers driven by the electrochemical potential. The detailed dynamic results showed that the chiral two-dimensional adlayers could be reversibly tuned purely by the applied electrode potential.

Phenomena associated with chirality have held a deep fascination for scientists since Pasteur manually separated left-handed and right-handed sodium ammonium tartrate crystals. ${ }^{1}$ It is one of the most attractive areas in chemistry because of the important applications in heterogeneous and asymmetric catalysis, ${ }^{2-5}$ enan-

\footnotetext{
* Corresponding author. Phone: +86-10-62849356. Fax: +86-10-62923563. E-mail: gjsu@rcees.ac.cn.

$\dagger$ Chinese Academy of Sciences.

* Research Center Jülich.

$\$$ Universidad Autonoma de Puebla.

(1) Pasteur, L. Ann. Chim. Phys. 1848, 24, 442-459.

(2) Wan, T. A.; Davies, M. E. Nature 1994, 370, 449-450.

(3) Jannes, G.; Dubois, V. Chiral Reaction in Heterogeneous Catalysis; Plenum: New York, 1995.

(4) Baiker, A.; Blaser, H. U. In Handbook of Heterogeneous Catalysis; Ertl, G., Knözinger, H.; Weitkamp, J., Eds.; VCH: Weinheim, 1997; Vol. 5, pp24222430 .
}

tiomeric selectivity, ${ }^{6}$ and chemical and pharmaceutical industries. ${ }^{7}$ In recent years, the study of the expression of the chirality on solid surfaces ${ }^{8-14}$ is receiving more and more attention, especially with the development of scanning tunnelling microscopy (STM), which is able to directly visualize the chirality at atomic/ submolecular resolution at various interfaces. On solid surfaces, chirality can be encountered by adsorption of chiral molecules or be induced through chiral ordering. For example, the racemic mixtures were found to spontaneously separate into chiral domains on $\mathrm{Cu}(111),{ }^{15} \mathrm{Au}(111),{ }^{8}$ and highly oriented pyrolytic graphite (HOPG).$^{10}$ The supramolecular chiral channels were formed by the chiral tartaric acids on a $\mathrm{Cu}(110)$ surface.${ }^{13}$ Furthermore, for simple geometrical reasons, three-dimensional (3D) achiral molecules may become two-dimensional (2D) chiral species upon adsorption due to the reduced symmetry at the surface. This case is called adsorption-induced chirality. ${ }^{16-19}$ Bernasek et al. observed the adsorption-induced chirality for 1-octadecanol on HOPG due to the mismatch between molecules and substrate. ${ }^{16}$ Barth and Kern et al. found the formation of $2 \mathrm{D}$ chiral assemblies for achiral 4-[trans-2-(pyrid-4-yl-vinyl)] benzoic acid (PVBA) on $\mathrm{Ag}$ $(111)^{17}$ and $\mathrm{Au}(111)^{18}$ via weak hydrogen bonds $(\mathrm{C}-\mathrm{H} \cdots \mathrm{O}$ and $\mathrm{C}-\mathrm{H} \cdots \mathrm{N})$. More recently, the results of a coverage-driven chiral phase transition for PVBA on $\mathrm{Cu}(100)$ have been reported. ${ }^{19}$

(5) Baiker, A. J. Mol. Catal. A: Chem. 1997, 115, 473-493.

(6) Maki, J. J.; Kauranen, M.; Persoons, A. Phys. Rev. B 1995, 51, 1425-1434.

(7) Sheldon, R. A. Chirotechnology; Marcel Dekker: New York, 1993.

(8) Yuan, Q. H.; Yan, C. J.; Yan, H. J.; Wan, L. J. J. Am. Chem. Soc. 2008, 130, 8878-8879.

(9) Humblot, V.; Barlow, S. M.; Raval, R. Prog. Surf. Sci. 2004, 76, 1-19.

(10) Elemans, J. A. A. W.; De Cat, I.; Xu, H.; De Feyter, S. Chem. Soc. Rev. 2009, 38, 722-736.

(11) Fasel, R.; Parschau, M.; Ernst, K.-H. Nature 2006, 439, 449-451.

(12) Pan, G. B.; Luo, J.; Deng, Q. Y.; Wan, L. J. J. Nanosci. Nanotechnol. 2008, 8, 5702-5707.

(13) Ortega Lorenzo, M.; Baddeley, C. J.; Muryn, C.; Raval, R. Nature 2000, 404, 376-379.

(14) Linares, M.; Iavicoli, P.; Psychogyiopoulou, K.; Beljonne, D.; Feyter, D.; Amabilino, D. B.; Lazzaroni, R Langmuir 2008, 24, 9566-9574.

(15) Xu, Q. M.; Wang, D.; Wan, L. J.; Wang, C.; Bai, C. L.; Feng, G. Q.; Wang, M. X. Angew. Chem., Int. Ed. 2002, 41, 3408.

(16) Cai, Y.; Bernasek, S. L. J. Am. Chem. Soc. 2004, 126, 14234-14238.

(17) Barth, J. V.; Weckesser, J.; Trimarchi, G.; Vladimirova, M.; De Vita, A.; Cai, C.; Brune, H.; Grünter, P.; Klaus, K. J. Am. Chem. Soc. 2002, 124, 7991-8000.

(18) Weckesser, J.; De Vita, A.; Barth, J. V.; Cai, C.; Kern, K. Phys. Rev. Let. 2001, 87, 0961011-096101-4.

(19) Vidal, F.; Delvigne, E.; Stepanow, S.; Lin, N.; Barth, J. V.; Kern, K. J. Am. Chem. Soc. 2005, 127, 10101-101106.

Analytical Chemistry, Vol. 81, No. 21, November 1, 2009

8741 
Homochiral ordered supramolecular nanostructures were found when the molecular coverage remained below a critical value. However, when the critical coverage was exceeded, an abrupt transition to a single racemic phase was observed.

On surfaces or at interfaces, the 2D phase behavior is mainly governed by a complex interplay between intermolecular and molecule-substrate interactions. ${ }^{20}$ In an electrochemical environment, the charge density at metal electrodes can be tuned by the external electric field, and it frequently steers the configuration and strength of adsorption and even results in the formation of different patterns. ${ }^{21-26}$ Wan et al. found the orientation transition of the 2,2 -bipyridine from flat-lying to vertical is completely potential dependent and reversible on $\mathrm{Cu}(111) .{ }^{22}$ Trimesic acid can reversibly form different patterns at different charge density at $\mathrm{Au}(111) / 0.1 \mathrm{M} \mathrm{HClO}_{4}$ or $\mathrm{Au}(111) / 0.05 \mathrm{M} \mathrm{H}_{2} \mathrm{SO}_{4}$ interfaces. ${ }^{25,26}$ Previous studies address that the electrochemical technique could control the formation of the nanostructure which presents a definitive milestone toward the development of a functional molecule element. However, to the best of our knowledge, the effect of the electric field on the expression of supramolecular chirality in two dimensions has not been reported up to now, although the chiral adlayers were successfully resolved in an electrochemical environment. ${ }^{15}$

In this work, the phase transition of chiral supramolecular adlayers of $p$-phenylenedi( $\alpha$-cyanoacrylicacid) di- $n$-ethyl ester ( $p$ CPAEt) has been investigated on $\mathrm{Au}(111)$ in $0.1 \mathrm{M} \mathrm{HClO}_{4}$ using electrochemical scanning tunneling microscopy (ECSTM) in combination with cyclic voltammetry. The $p$-CPAEt molecule is a typical diolefinic compound. ${ }^{27}$ The structural scheme is shown in Figure 1a, and the optimized geometry ${ }^{28}$ is shown in Figure $1 \mathrm{~b}$ along with the illustration of two-dimensional (2D) chiral species designated $\lambda$ - $p$-CPAEt and $\delta$ - $p$-CPAEt existing at a surface. For the transformation of the $\lambda$ - into the $\delta$-species, a mirror operation is mandatory. Similar surfaced-induced 2D chiral molecules have been reported by Barth and Kern et al. ${ }^{17-19}$ Characteristically, the $p$-CPAEt molecule is a $\pi$-conjugated planar molecule with an aromatic ring, two olefinic double bonds, two ethyl ester groups, and two cyanoic polar groups. It can be easily judged that this molecule can provide the functional groups $\mathrm{N} \equiv \mathrm{C}-$ and $\mathrm{C}=\mathrm{O}$ for potential hydrogen bonds. The obtained results showed that three types of chiral supramolecular nanostructures, formed by $2 \mathrm{D}$ chiral $p$-CPAEt species via the intermolecular interactions, were resolved at differently charged interfaces. It is very interesting that different chiral nanopatterning on the solid surfaces could be reversibly tuned by the applied electrode

(20) Cyganik, P.; Buck, M.; Wilton-Ely, J. D. E.; Wöll, C. J. Phys. Chem. B 2005, 109, 10902-10908.

(21) Zhang, J. D.; Kuznetsov, A. M.; Medvedev, I. G.; Chi, Q. J.; Albrecht, T.; Jensen, P. S.; Ulstrup, J. Chem. Rev. 2008, 108, 2737-2791.

(22) Wan, L. J.; Noda, H.; Wand, C.; Bai, C. L.; Osawa, M. ChemPhysChem 2001, 10, 617-619.

(23) Su, G. J.; Zhang, H. M.; Wan, L. J.; Bai, C. L. Surf. Sci. 2003, 531, L363L368.

(24) Cunha, F.; Tao, N. J.; Wang, X. W.; Jin, Q.; Duong, B.; D’Agnese, J. Langmuir 1996, 12, 6410-6418.

(25) Su, G. J.; Zhang, H. M.; Wan, L. J.; Bai, C. L.; Wandlowski, Th. J. Phys. Chem. B 2004, 108, 1931-1937.

(26) Li, Z.; Han, B.; Wan, L. J.; Wandlowski, Th. Langmuir 2005, 21, 69156928.

(27) Nakanishi, F.; Hasegawa, M. J. Polym. Sci. 1970, 8, 2151-2160.

(28) Wu, P.; Zeng, Q.-D.; Wang, C.; Bai, C. L. Surf. Interface Anal. 2001, 32, 262-265. (a)

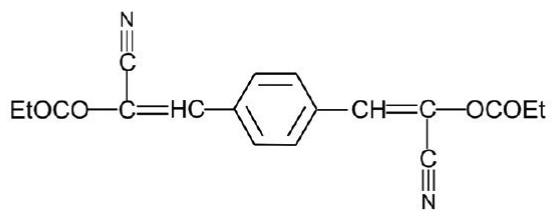

(b)

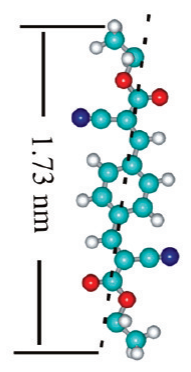

$\lambda$-p-CPAEt

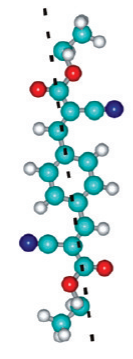

$\delta$-p-CPAEt

Figure 1. (a) Structural formula of $p$-CPAEt. (b) Space-filling models of $p$-CPAEt molecule with the definition of the molecular axis marked by dashed line. The surface-induced 2D chiral species are labeled as $\lambda$ - and $\delta$ - $p$-CPAEt.

potential. This is the first report of the effect of the external electric field on the chiral adlayers. We hope that the obtained results could provide the important information from the fundamental and applied branches of material sciences.

\section{EXPERIMENTAL SECTION}

Chemicals and Monolayer Preparation. Electrolyte solutions were prepared with Milli-Q water $(18 \mathrm{M} \Omega$, TOC $\leq 3 \mathrm{ppb})$, $\mathrm{HCl}$ (Merck, suprapure), and $\mathrm{HClO}_{4}$ (from Kanto Chemical Co. of Tokyo or from Merck, suprapure). Absolute ethanol was purchased from KMF Laborchemie Handels GmbH (Germany). $p$-CPAEt was prepared and recrystallized as described in an earlier paper. ${ }^{27,28}$

The film was prepared by immersing the gold single crystals into saturated $p$-CPAEt-ethanol solution for $60 \mathrm{~s}$. Then, it was rinsed successively with ethanol and water so that excess molecules were gently removed.

Electrochemical Experiments. The electrochemical measurements were performed with an Autolab PGSTAT 30 workstation employing a home-built 3-electrode glass cell in $0.1 \mathrm{M} \mathrm{HClO}_{4}$ at room temperature. The electrolyte was deaerated with argon before and during the measurements. The counter electrode was a platinum wire, and a trapped-hydrogen electrode served as reference. The ideal $\mathrm{Au}$ (111) electrodes were single-crystal cylinders of $4 \mathrm{~mm}$ height and $4 \mathrm{~mm}$ diameter. Contact with the electrolyte was always established under strict potential control in a hanging meniscus configuration.

Scanning Tunneling Microscopy. The STM experiments were carried out in $0.1 \mathrm{M} \mathrm{HClO}_{4}$ with Nanoscope E (Digital Instruments) and Molecular Imaging Pico SPM. The tunneling tips were prepared by electrochemical etching of a tungsten wire $(0.25 \mathrm{~mm}$ in diameter), subsequently coated with clear nail polish or polyethylene to minimize Faradaic currents. An additional checking experiment was performed in argon box with electrochemically etched Pt/Ir tip (70\%/30\%, $0.25 \mathrm{~mm}$ ). The Au(111) electrodes for the film preparation were obtained with the bead method by melting a Au wire (99.999\% in purity, $0.8 \mathrm{~mm}$ in diameter) or commercial disk-shape $\mathrm{Au}(111)$ massive 


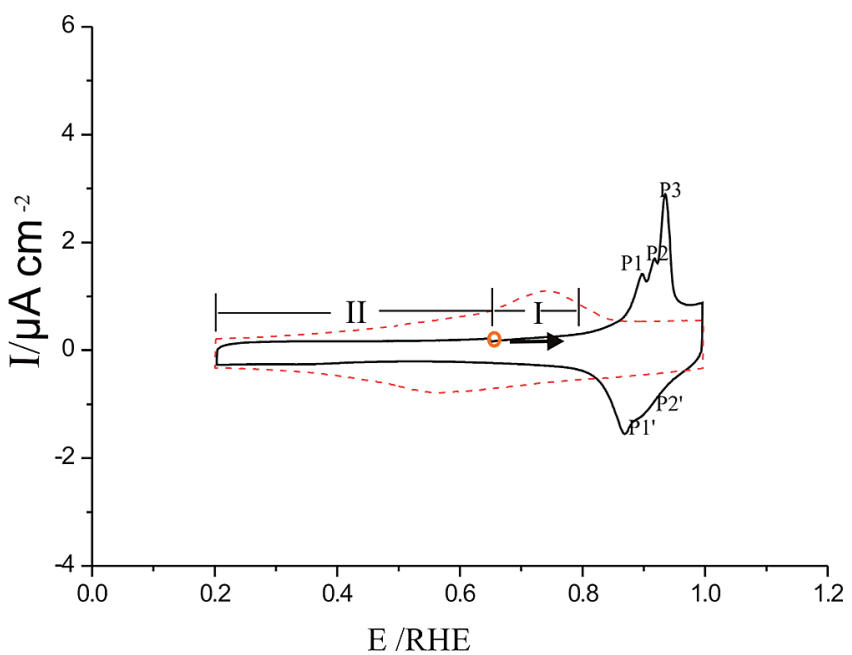

Figure 2. Current vs potential curves for $\mathrm{Au}(111)$ electrode $/ 0.1 \mathrm{M}$ $\mathrm{HClO}_{4}$ in the absence (red dashed line) and in the presence of $p$-CPAEt film with first positive scanning (solid trace). $E_{\text {start }}=0.65 \mathrm{~V}$, scan rate $10 \mathrm{mV} \mathrm{s}^{-1}$.

single crystals (10 mm diameter, $2 \mathrm{~mm}$ height). The unreconstructed surfaces were achieved by the same method as described above. All STM images were acquired in constant current mode at room temperature. Platinum wires served as quasireference and counter electrodes. In this paper, all electrode potentials are quoted with respect to a reversible hydrogen electrode (RHE).

\section{RESULTS AND DISCUSSION}

Steady-State Voltammetry. Figure 2 shows the typical current versus potential curves of bare $\mathrm{Au}(111)$ (dashed line) ${ }^{29,30}$ and the $p$-CPAEt film on the $\mathrm{Au}(111)$ (solid line) in $0.1 \mathrm{M} \mathrm{HClO}_{4}$ recorded at a scan rate of $10 \mathrm{mV} \mathrm{s}^{-1}$. It can be seen that the presence of the $p$-CPAEt molecules causes a slight decrease of the capacitance. The solid trace in Figure 2 represents the first positive-going voltammetric cycle after the equilibration in the double layer region at the starting potential of $0.65 \mathrm{~V}$. The current peaks ( $\mathrm{P} 1\left|\mathrm{P} 1^{\prime}, \mathrm{P} 2\right| \mathrm{P} 2$ ', and $\left.\mathrm{P} 3\right)$ have been resolved around $0.9 \mathrm{~V}$. According to the reported literature, ${ }^{31,32}$ they were expected to be very complex as in the case of 4-cyanopyridine on metal electrodes, where the hydrolysis of the cyano group may occur at highly positive potentials. If the potential range is negatively extended to $E<0.2 \mathrm{~V}$, the reductive peaks will appear just before the onset of hydrogen evolution and the $p$-CPAEt molecules may be reduced. ${ }^{32}$ The detailed understanding of these results requires a qualitative and quantitative product analysis based on spectroscopic experiments, which is beyond the scope of the present work. Our interest focuses on investigating the adlayer structures of the $p$-CPAEt film in the potential double layer region from 0.2 to $0.8 \mathrm{~V}$. In this range, two characteristic potential regions have been defined (labeled I, II,), with the boundary point divided from the starting scanning potential. In the positive direction, region I is extended to be $0.8 \mathrm{~V}$ just before the appearance of the current

(29) Honbo, H.; Sugawara, S.; Itaya, K. Anal. Chem. 1990, 62, 2424-2429.

(30) Angerstein-Kozlowska, H.; Conway, B. E.; Hamelin, A.; Stoicoviciu, L. J. J. Electroanal. Chem. 1987, 228, 429-453.

(31) Richer, J.; Lipkowski, J. J. Electrochem. Soc. 1986, 133, 121-128.

(32) Pluchery, O.; Climent, V.; Rodes, A.; Tadjeddine, A. Electrochim. Acta 2001, $46,4319-4329$

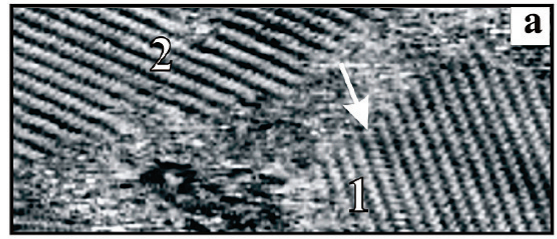

$20 \mathrm{~nm} \times 50 \mathrm{~nm}$
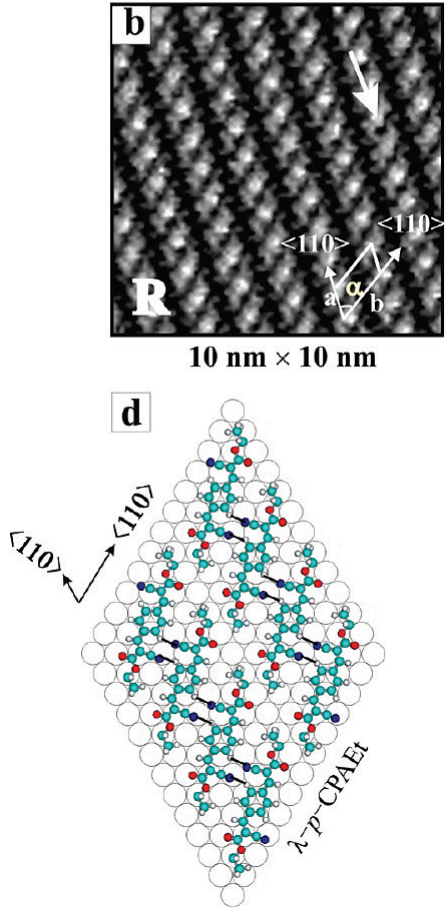

$10 \mathrm{~nm} \times 10 \mathrm{~nm}$

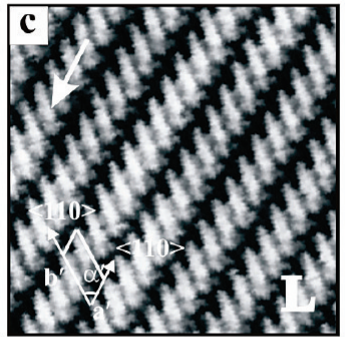

$10 \mathrm{~nm} \times 10 \mathrm{~nm}$

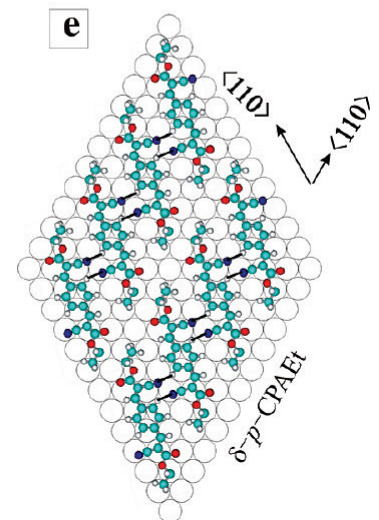

Figure 3. (a) Large-scale STM image of the stripe pattern on $\mathrm{Au}(111)$ in $0.1 \mathrm{M} \mathrm{HClO}_{4}(20 \mathrm{~nm} \times 50 \mathrm{~nm})$. (b, c) High-resolution STM images $(10 \mathrm{~nm} \times 10 \mathrm{~nm})$ of $R$ and $L$ domains. $E_{\mathrm{s}}=0.71 \mathrm{~V}$. $(\mathrm{d}, \mathrm{e})$ Tentatively suggested structural models for $\lambda$-phase and $\delta$-phase of stripe pattern.

peaks ( $\mathrm{P} 1\left|\mathrm{P} 1^{\prime}, \mathrm{P} 2\right| \mathrm{P} 2^{\prime}$, and $\left.\mathrm{P} 3\right)$ whereas region II is negatively delimited by $0.2 \mathrm{~V}$ to avoid the reduction of $p$-CPAEt molecules. When the scan rate of the cyclic voltammogram was reduced to $3 \mathrm{mV} \mathrm{s}^{-1}$, one pair of extremely weak phase transition peaks was observed around $0.65 \mathrm{~V} \mathrm{RHE}$ as shown by the cyclic voltammogram in Figure S1, indicating that a very slow phase transition existed between regions I and II. In the following section, the potential-induced phase transition of $p$-CPAEt adlayer on $\mathrm{Au}(111)$ was discussed between potential regions I and II. Here, we place emphasis on the phase transitions purely driven by changes of the electrode potential and not by redox-modifications of the adsorbed species. Similar phenomena were reported for porphyrine on $\mathrm{Cu}(111)^{33}$ and for 4-methylbenzenethiol on $\mathrm{Au}(111){ }^{34}$

In Situ STM Studies. Stripe Pattern (Region I, $0.65 \mathrm{~V} \rightarrow 0.8$ $V)$. A freshly prepared $p$-CPAEt film on $\mathrm{Au}(111)$ was directly transferred into the electrochemical STM cell in contact with 0.1 $\mathrm{M} \mathrm{HClO}_{4}$ electrolyte solution at the open circuit potential (OCP) around $0.71 \mathrm{~V}$. As shown in Figure 3a, the long-range ordered stripe patterns were observed on the large terrace of $\mathrm{Au}(111)$

(33) Hai, N. T. M.; Gasparovic, B.; Wandelt, K.; Broekmann, P. Surf. Sci. 2007, 601, 2597-2602.

(34) Seo, K.; Borguet, E. J. Phys. Chem. C 2007, 111, 6335-6342. 
surface with parallel bright ridges and dark troughs. Two domains, 1 and 2, were separated by a fuzzy domain boundary.

High resolution STM images for domains 1 and 2 are shown in Figure 3b,c, respectively. It was observed that each $p$-CPAEt molecule adsorbed on $\mathrm{Au}(111)$ surface by linear configuration. The central bright rods corresponded to the conjugated backbone of $p$-CPAEt molecules. The central brightest part was attributed to a phenyl ring. Its width is $\sim 0.57 \mathrm{~nm}$ and comparable to the diameter of a phenyl ring, suggesting a planar surface orientation. The cross-section measurements in Figure S2 show the apparent corrugation height of the core spots was between 0.1 and 0.15 $\mathrm{nm}$, corresponding to typical values for physisorbed aromatic molecules with their $\pi$ system parallel to the substrate surface..$^{35,36}$ On the basis of these results, it could be concluded that $p$-CPAEt molecules might take the planar orientation via the interaction between the conjugated $\pi$ system and substrate in the stripe pattern. A similar conclusion on the molecular orientation was reported for trimesic acid and isophthalic acid on $\mathrm{Au}(111) .^{26,37}$ According to the repeating of the molecular arrays, the rhombus unit cells were outlined in both images and the characteristic dimensions were summarized in Table S1. In comparison with the hexagonal lattice of $\mathrm{Au}(111)$, the directions $a / a^{\prime}$ and $b / b^{\prime}$ are parallel to the close-packed direction $\langle 110\rangle$ of the surface. A $(3 \times$ 6) structure containing one molecule can be defined for both parts $\mathrm{b}$ and $\mathrm{c}$ of Figure 3, giving rise to a coverage $\Gamma=1.27 \times 10^{-10}$ $\mathrm{mol} \mathrm{cm}{ }^{-2}$. However, after careful observation, it was found that, in the direction indicated by the bold white arrows, the next molecule shifts in clockwise orientation in Figure $3 \mathrm{~b}$ but anticlockwise orientation in Figure 3c. Both stripe structures cannot be superimposed simply by the translation and rotation within the surface plane although they have the same characteristic dimensions within errors. Therefore, two chiral domains were formed. They were designated right-turning (R) and left-turning (L), respectively. Similar chiral stripe features have been found for 3,5-di(4- $n$-tetradecyloxphenlyl) pyrazole (DTPP) on graphite surface. ${ }^{38}$ In addition, it is observed that one mirror domain is frequently intermediated by one or two opposite enantiomorphous rows as marked by the bold white arrow in Figure 3a.

On the basis of the molecular arrangement and chirality as well as the intermolecular interaction such as the hydrogen bonding, structural models were proposed in Figure 3d,e for R and $\mathrm{L}$ chiral assemblies. The chiral motifs of $\mathrm{L}$ and $\mathrm{R}$ domains were built respectively by the adsorption-induced chiral $\lambda$-p-CPAEt and $\delta$-p-CPAEt, which is more reasonable than vice versa. The benzene rings were closely fixed in the three hollow sites of $\mathrm{Au}(111)$ surfaces via $d-\pi^{*}$ coordination between the molecule and substrate. ${ }^{35,36}$ The van der Waals interaction exists between the molecular rows. The lateral positions of neighboring molecules in each row are mainly controlled by the $\mathrm{C}-\mathrm{H} \cdots \mathrm{N} \equiv \mathrm{C}$ bond. The mirror positioning of the $-\mathrm{C} \equiv \mathrm{N}$ groups switch the mirror alignment of $p$-CPAEt lamellae on the $\mathrm{Au}(111)$ surface via lateral weak hydrogen bonding. The distance of the $\mathrm{C}-\mathrm{H} \cdots \mathrm{N} \equiv \mathrm{C}$ bond is estimated to be spatially $0.21 \mathrm{~nm}$, comparable to those found in other systems with weak hydrogen bonding interactions

(35) Chiang, S. Chem. Rev. 1997, 97, 1083-1096.

(36) Sautet, P. Chem. Rev. 1997, 97, 1097-1116.

(37) Li, Z.; Wandlowski, Th. J. Phys. Chem. C 2009, 113, 7821-7825.

(38) Li, C. J.; Zeng, Q. D.; Wang, C.; Wan, L. J.; Xu, S. L.; Wang, C. R.; Bai, C. L. J. Phys. Chem. B 2002, 106, 13262-13267. responsible for lamellae arrangements. ${ }^{19,39,40}$ This type of weak hydrogen bonds has been extensively confirmed by theoretical computation $^{41,42}$ and crystal structural analysis of the organic molecules with cyano groups. ${ }^{39,40}$ It takes an important role in supramolecular engineering ${ }^{19,43}$ although the $\mathrm{C}-\mathrm{H} \cdots \mathrm{N}$ has a relatively weak interaction in comparison with $\mathrm{O}-\mathrm{H} \cdots \mathrm{N}$ and $\mathrm{O}-\mathrm{H} \cdot \cdots \mathrm{O}$. This type of chiral arrangement could be assigned to the adsorption of $2 \mathrm{D}$ chiral $p$-CPAEt and its $2 \mathrm{D}$ extension in the molecular assemblies via the intermolecular interactions. Similar results were reported by Barth and Kern et al. for 4-[trans-2-(pyrid4-yl-vinyl)] benzoic acid on $\mathrm{Ag}(111)$ and $\mathrm{Au}(111)$ and $\mathrm{Cu}(100) .{ }^{17-19}$ Additionally, the arrangement for the defect row pointed by the arrow in Figure $3 \mathrm{a}$ is similar to that in Figure 3c. It is formed by $\delta$ - $p$-CPAEt species and could be presented by the model row in Figure 3e. The intermediation of the reverse chiral defect row may result from the weak van der Waals interaction between the molecular rows.

Moreover, scanning the electrode potential from 0.65 to 0.8 $\mathrm{V}$, no additional phases were found except for the stripe pattern. For the observed stripe patterns, we wondered if they were directly formed by the deposition of $p$-CPAEt on a $\mathrm{Au}(111)-(1 \times$ 1) electrode from the saturated sample-ethanol solution. This assumption was confirmed by the experiments carried out in argon atmosphere without the electrochemical potential control where the two same enantiomeric stripe patterns have been clearly observed within the experimental error.

Network Patterns (Region II, $0.2 \rightarrow 0.65 \mathrm{~V}$ ). Scanning the electrode potential in the region $0.2<E \leq 0.65 \mathrm{~V}$, a rather different STM contrast appears on the electrode surface accompanied by the dissolution of the stripe phases. After the dynamic equilibration, the steady-state STM images are measured (Figure 4). As showed in Figure 4a, four different domains $\left(1,1^{\prime}, 2,3\right)$ could be distinguished, appearing structurally inhomogeneous. Domain 1 , and its translational domain $1^{\prime}$, and domain 2 are denoted as network I whereas domain 3 is denoted as network II. It seems that reconstructed surface patches preferentially support network structure I, but on unreconstructed surface patches of network structure II are more welcomed.

For network I, Figure 4b,c represents the typical highresolution STM images from domains 1 and 2, respectively. Each recognized bright rod was assigned as an individual $p$-CPAEt molecule. Intriguingly, four $p$-CPAEt molecules formed a tetramer with the triangular cloverleaf clusters marked by the black curve arrows, attached with one extra linked bright rod. The latter formed the double unit with one parallel molecule from the former. Careful observation could reveal that there were two types of tetramers, clockwise fashion in Figure $4 \mathrm{~b}$ and anticlockwise in Figure 4c. Therefore, two chiral domains were formed in the molecular assembly in which the chiral tetramer were an essential

(39) Kumar, S.; Koellner, G.; Steiner, Th.; Subramanian, K. J. Chem. Crystallogr. 1998, 28, 149-152.

(40) Thaimattam, R.; Shekhar Reddy, D.; Xue, F.; Mak, Th.C.W.; Nangia, A.; Desiraju, G. J. Chem. Soc., Perkin Trans. 2 1998, 178, 3-1788.

(41) Coussan, S.; Bouteiller, Y.; Perchard, J. P.; Brenner, V.; Millié, P.; Zheng, W. Q.; Talbot, F. J. Chem. Phys. 1999, 110, 10046-10057.

(42) Okuno, Y.; Yokoyama, T.; Yokoyama, S.; Kamikado, T.; Mashiko, S. J. Am. Chem. Soc. 2002, 124, 7218-7225.

(43) Meier, C.; Ziener, U.; Landfester, K.; Weihrich, P. J. Phys. Chem. B 2005, 109, 21015-21027. 


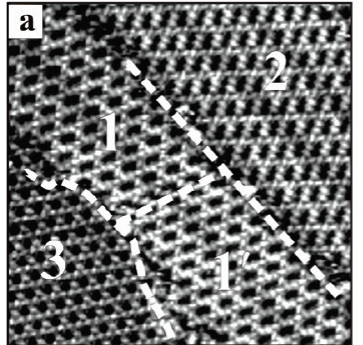

$40 \mathrm{~nm} \times 40 \mathrm{~nm}$

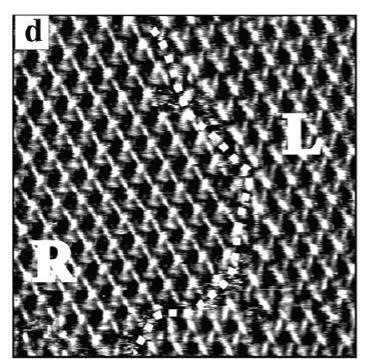

$30 \mathrm{~nm} \times 30 \mathrm{~nm}$

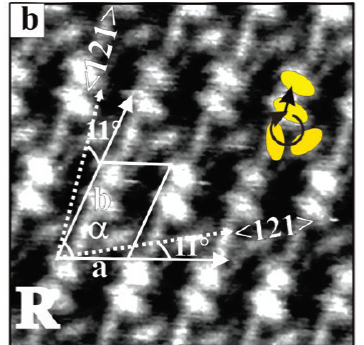

$10 \mathrm{~nm} \times 10 \mathrm{~nm}$

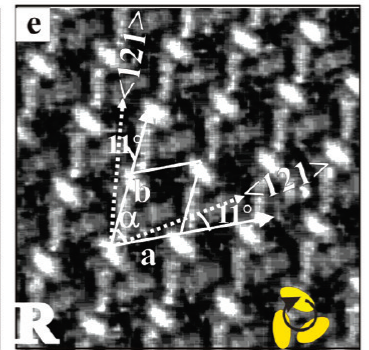

$10 \mathrm{~nm} \times 10 \mathrm{~nm}$

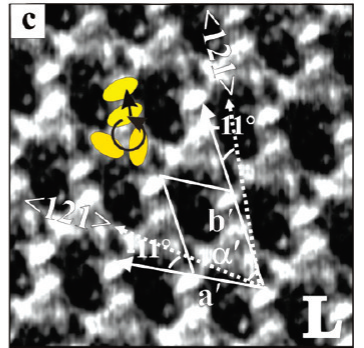

$10 \mathrm{~nm} \times 10 \mathrm{~nm}$

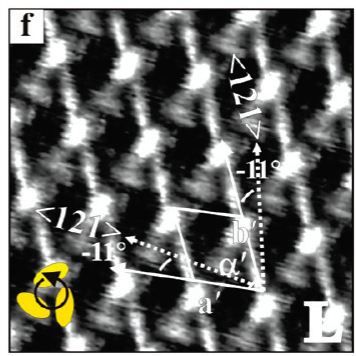

$10 \mathrm{~nm} \times 10 \mathrm{~nm}$

Figure 4. (a) Large-scale STM image $(40 \mathrm{~nm} \times 40 \mathrm{~nm})$ of $p$-CPAEt film on $\mathrm{Au}(111)$ in $0.1 \mathrm{M} \mathrm{HClO}_{4}$ showing the coexistence of network I and II. (b, c) High-resolution STM images $(10 \mathrm{~nm} \times 10 \mathrm{~nm}$ ) of R and L domains for network I. (d) Large-scale STM image $(25 \mathrm{~nm} \times 25 \mathrm{~nm})$ of network II. $(e, f)$ Close-up STM images $(10 \mathrm{~nm} \times 10 \mathrm{~nm})$ of $R$ and $L$ domains for network II. For all the images from a to $f, E_{s}$ is equal to 0.55 V.

chiral unit but with different rotation. Figure $4 \mathrm{~b}, \mathrm{c}$ could be designated for $\mathrm{R}$ domain and $\mathrm{L}$ domain, respectively. On the basis of the periodicity of the molecular arrays, a unit cell containing four molecules can be outlined and measured in both parts $b$ and $\mathrm{c}$ of Figure 4. All the directions along the vectors ( $\mathrm{a}$ and $\mathrm{b}$ or $\mathrm{a}^{\prime}$ and $b^{\prime}$ ) were parallel to the $\sqrt{ } 7$ direction of the substrate surface, and tilted by the opposite sign of $\sim 11^{\circ}$ with $\langle 121\rangle$ of the substrate in both chiral domains. It means that the organic adlayer does not strictly follow the main symmetric directions of the $\mathrm{Au}(111)$ surface. A similar mismatch between the orientation of chiral adlayers and one of the main symmetry axes or the axes that run normal to them on the substrate underneath were reported in the literature. ${ }^{10,19}$ The characteristic dimensions of unit cells were summarized in Table S1. The $(3 \sqrt{ } 7 \times 4 \sqrt{ } 7)$ structures could be defined for two chiral domains with the surface coverage of 1.10 $\times 10^{-10} \mathrm{~mol} \mathrm{~cm}^{-2}$ which was smaller than that for the stripe pattern.

The observations show that network II also has the mirror property. Figure 4d represents a typical STM image containing two chiral domains separated by mirror domain boundaries. Figure 4e,f shows the corresponding close-up STM images for R and L domains, respectively. It can be seen that the network structures were purely built up by the trimer with the triangular cloverleaf clusters marked by black curve arrow. The molecules in triangular cloverleaf clusters in Figure 4e seem to rotate exclusively clockwise while in Figure 4f, the molecules were exclusively arranged in an anticlockwise fashion. The experimentally determined rhombohedral until cell containing three $p$-CPAEt molecules was outlined in both images. All the directions along the vectors ( $a$ and $b$ or $a^{\prime}$ and $b^{\prime}$ ) were parallel to the $\sqrt{ } 7$ direction of the substrate surface. The characteristic dimensions of unit cells were summarized in Table S1. The $(3 \sqrt{ } 7 \times 3 \sqrt{ } 7)$ structures could be defined for two chiral domains. The surface coverage was equal to that of network I, but both were smaller than that of the stripe pattern.
According to the information obtained from STM experiments and chirality, we try to construct the structural models for both types of networks I and II. Previous studies address that molecules with $\mathrm{N} \equiv \mathrm{C}-$ groups easily form dimers and trimers via weak hydrogen bonds. ${ }^{28,44,45}$ On the basis of these literature reports and the present experimental data, the adsorption models of network I and II were tentatively suggested in Figure 5a,b and Figure $5 c, d$, respectively. The chiral motifs of $\mathrm{R}$ and $\mathrm{L}$ domains for both networks I and II were built up by the adsorption-induced chiral $\lambda$-p-CPAEt and $\delta$ - $p$-CPAEt, respectively. The benzene rings occupy the position as close to the three hollow sites as possible. The triangular cloverleaf clusters marked by the black curve arrows were induced by the weak hydrogen bond between $\mathrm{H}$ atoms and $\mathrm{C} \equiv \mathrm{N}-$. The distances varied from 0.20 to $0.27 \mathrm{~nm}$, within the typical range of weak hydrogen bonds. ${ }^{19,39,40}$ For network II, it can be seen that an extended hydrogen network exists on the overall domain (Figure $5 c, d$ ). However, the additional interaction exists between one extra linked $p$-CPAEt molecule and triangular cloverleaf clusters for network I (Figure 5a,b). The $\mathrm{H}$ atoms in $\mathrm{H}_{2} \mathrm{O}$ molecules may form hydrogen bonds together with $\mathrm{N}(\mathrm{CN})$ and $\mathrm{O}(\mathrm{CO})$ groups, which helps the one extra molecule forming double units with one parallel molecule from triangular cloverleaf clusters. From the models in Figure 5a,b and Figure 5c,d, it can be judged that the mirror positions of $-\mathrm{C} \equiv \mathrm{N}$ would lead to a switch in supramolecular assembly directions.

Structural Transitions and Dynamic Processes. The steady-state STM experiments in an electrochemical environment demonstrated that depending on the substrate charge density, three types of ordered chiral supramolecular adlayers can be observed on a $\mathrm{Au}$ (111) surface. We also notice that these chiral nanostructures

(44) Yokoyama, T.; Yokoyama, S.; Kamikado, T.; Okuno, Y.; Mashiko, S. Nature 2001, 413, 619-621.

(45) Spillmann, H.; Kiebele, A.; Stöhr, M.; Jung, Th. A.; Bonifazi, D.; Cheng, F.; Diedrich, F. Adv. Mater. 2006, 18, 275-279. 

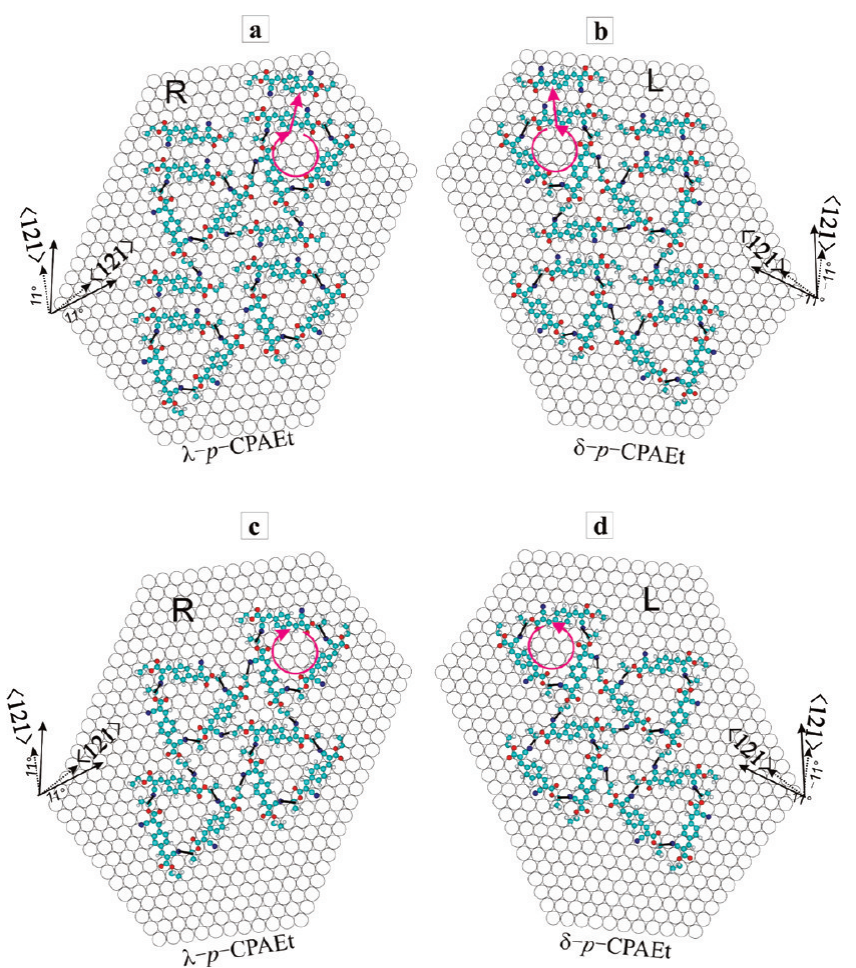

Figure 5. (a, b) Suggested adsorption models for $R$ and $L$ domains of network I. (c, d) Suggested adsorption models for $R$ and $L$ domains of network II.

can be reversibly dissolved or formed by tuning the electrode potential. The transition of the stripe adlayers toward the network phases was accompanied theoretically by the reduction of the surface coverage from $1.29 \times 10^{-10} \mathrm{~mol} \mathrm{~cm}^{-2}$ to $1.10 \times 10^{-10} \mathrm{~mol}$ $\mathrm{cm}^{-2}$ according to the suggested models for $p$-CPAEt on $\mathrm{Au}$ (111). The coverage changes very little during the transition. It is a little higher in the potential region $0.65 \mathrm{~V}<E<0.8 \mathrm{~V}$ than in the potential region $0.2 \mathrm{~V}<E \leq 0.65 \mathrm{~V}$. In this section, we focus on addressing the dynamic changes among these chiral supramolecular nanostructures.

Stripe Pattern $\rightarrow$ Network Pattern in Potential Region II. If newly prepared $p$-CPAEt films were transferred into the electrochemical cell under potential control at $0.2 \mathrm{~V}<E<0.65 \mathrm{~V}$ (region II), i.e., within the thermodynamically stable range of network structures, the deposited ordered stripe patterns are observed initially. However, these stripe patterns are not thermodynamically stable in this potential region. Over time, these structures began dissolving into a disordered phase from the defects of the substrate lattice and most frequently from the domain boundary. The reason might be that the adsorbed species on these local sites have a higher energy due to fewer numbers of hydrogen bonds for $p$-CPAEt molecules at the end of the domain. Meanwhile, the new network patterns evolve via the rearrangement of $p$-CPAEt molecules and its long-range order increases with observation time. A snapshot of this process is presented in Figure $\mathrm{S} 3$, and the evolution of the structural changes is monitored as a function of time. The experiment starts with the newly prepared $p$-CPAEt film on the reconstructed $\mathrm{Au}(111)-(\mathrm{p} \times \sqrt{ } 3)$ which is directly transferred into the electrochemical STM cell at $0.58 \mathrm{~V}$. Initially, highly ordered stripe patterns have been observed on the gold surface. However, after $3-7 \mathrm{~h}$, the fuzzy domain boundaries appear to broaden (Figure S3a), which is accompanied with the disintegration of the stripe phase. Through the intermediate liquidlike $p$-CPAEt phase with a distinct local order, a rather new network STM-contrast pattern starts to evolve (Figure S3b). The close-up STM images in Figure S3c clearly resolve the removal of single $p$-CPAEt molecule from the stripe domain. The rate of the transformation process is very fast once it starts and is easily promoted by tip scanning, reduced tunneling resistance, and even small potential step. This suggests a high mobility of $p$-CPAEt monomers and a rather weak adsorbate-substrate corrugation potential in this region for the stripe pattern. The overall process from part a of Figure S3 to part d reveals that a sufficient negative polarization of the electrode surface can result in the formation of network patterns which follow a nucleation and growth mechanism.

We note that the same transforming phenomena are also observed on the unreconstructed $\mathrm{Au}(111)$ surface. The only difference is that the stable time of the stripe pattern is a little shorter $(1-3 \mathrm{~h})$ than that on $\mathrm{Au}(111)-(\mathrm{p} \times \sqrt{ } 3)$. This means that the corrugated reconstructed lines can block the dissolution of the stripe adlayer. One example is given in Figure S4 where the unreconstructed surface is decorated by the reconstruction lines. The step in the lower corner is used as a positional marker. It can be seen that the stripe patterns on $\mathrm{Au}(111)-(1 \times 1)$ have been already completely dissolved into the fluidlike transient disordered phase and the network structures come out on the local area. However, the stripe pattern, with domain boundary marked by a white arrow, occupies the whole reconstruction line (Figure S4a). Over time, network pattern evolves from the domain boundary and increase in size as shown in Figure S4b,c. The boundary between new network and two original stripe domains are marked by lateral white arrows. Finally, the stripe pattern is completely transferred into the network pattern as shown in Figure S4d. Looking through the overall sequence, it is obvious that the transforming process is delayed by a reconstruction line.

Dissolution of Network Pattern at $0.2 \mathrm{~V}$. After obtaining the stable network structure, and stepping the electrode potential negatively, one can observe that the network patterns start to dissolve at $0.2 \mathrm{~V}$ (Figure S5). The active sites are evenly distributed across entire terraces without specific preference to defects of the adlayer or substrate lattice. The dissolution grows rather isostropically with time. The overall process is delayed by the reconstruction line. It means that not only the reformation but also the potential-induced dissolution of the network patterns are significantly inhibited by the presence of the reconstruction lines.

Stripe Pattern $\rightarrow$ Network Pattern in Potential Region II $\rightarrow$ Stripe Pattern in Potential Region I. Figure 6 shows the detailed dynamic changes of three types of chiral supramolecular nanostructures tuned by the applied electric field. The experiment started with a freshly prepared $p$-CPAEt film which is transferred into the electrochemical STM cell at $0.55 \mathrm{~V}$ within the thermodynamically stable region of network patterns. The monitored spot is selected by an area with coexistence of the deposited stripe structure, from the sample-ethanol solution, and the evolved network structure (Figure 6a). It means that some patches of the stripe pattern were already tuned into the network structures after $\mathrm{Au}$ (111) electrode contacted with electrolyte within thermodynamically stable po- 


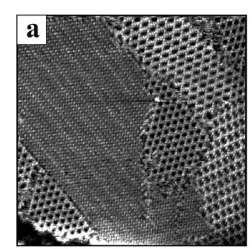

$60 \mathrm{~nm} \times 60 \mathrm{~nm}$

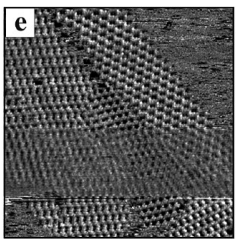

$60 \mathrm{~nm} \times 60 \mathrm{~nm}$

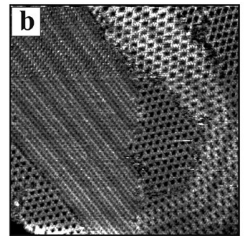

$60 \mathrm{~nm} \times 60 \mathrm{~nm}$

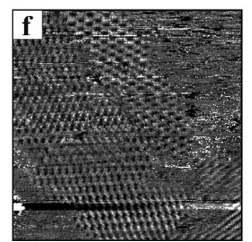

$60 \mathrm{~nm} \times 60 \mathrm{~nm}$

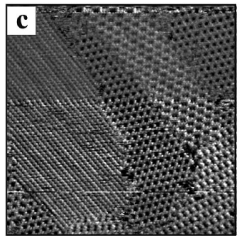

$60 \mathrm{~nm} \times 60 \mathrm{~nm}$

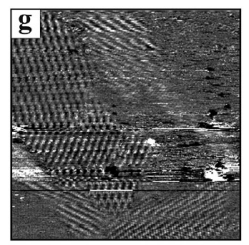

$60 \mathrm{~nm} \times 60 \mathrm{~nm}$

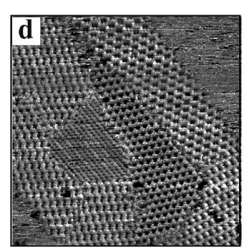

$60 \mathrm{~nm} \times 60 \mathrm{~nm}$

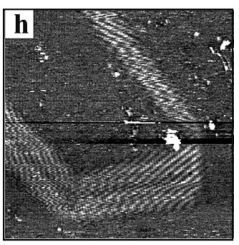

$60 \mathrm{~nm} \times 60 \mathrm{~nm}$

Figure 6. Potential-dependent series of STM images from $E_{\mathrm{s}}=0.55 \mathrm{~V}$ to $E_{\mathrm{s}}=0.75 \mathrm{~V}$ : (a) $t=0 \mathrm{~s}, E_{\mathrm{s}}=0.55 \mathrm{~V}$; (b) $t=253 \mathrm{~s}, E_{\mathrm{s}}=0.6 \mathrm{~V}$; (c) $t=896 \mathrm{~s}, E_{\mathrm{s}}=0.65 \mathrm{~V}$; (d) $t=1003 \mathrm{~s}, E_{\mathrm{s}}=0.65 \mathrm{~V}$; (e) $t=1172 \mathrm{~s}, E_{\mathrm{s}}=0.65 \mathrm{~V}$; (f) $t=1503 \mathrm{~s}, 0.67 \mathrm{~V} ;(\mathrm{g}) t=1678 \mathrm{~s}, E_{\mathrm{s}}=0.7 \mathrm{~V} ;(\mathrm{h}) t=2478 \mathrm{~s}$, $E_{\mathrm{s}}=0.75 \mathrm{~V}$.

tential region of network patterns. Scanning the electrode potential positively at $10 \mathrm{mV} \mathrm{s}^{-1}$, but within the thermodynamic stability range of the network structure, we found that the stripe nanostructure was gradually decaying and network nanostructures were enlarging (network I on $\mathrm{Au}(111)-(\mathrm{p} \times \sqrt{ } 3)$ and network II on $\mathrm{Au}(111)-(1 \times 1)$, Figure $6 \mathrm{~b}-\mathrm{d})$. At $E=0.65 \mathrm{~V}$ (Figure 6e), the stripe pattern was completely transferred into the network structure, but simultaneously, it is also noted that the network structures began to dissolve at $0.65 \mathrm{~V}$ from the upper right corner. This suggests that network nanostructures are not stable on less negatively charged electrodes. With continuing to increase the electrode potential positively until $0.67 \mathrm{~V}$ (Figure 6f), the stripe pattern appears locally on the reconstruction lines where the active role of the $\mathrm{Au}(111)-(\mathrm{p} \times \sqrt{ } 3)$ reconstruction elements was preferred as nucleation sites. ${ }^{18} \mathrm{~A}$ similar process was found for 2,2'-bipyridne on $\mathrm{Au}(111)$ in $0.05 \mathrm{M} \mathrm{H}_{2} \mathrm{SO}_{4} \cdot{ }^{22}$ Unfortunately, the creation of a true equilibrium pattern of the ordered $p$-CPAEt stripe pattern in $0.1 \mathrm{M} \mathrm{HClO}_{4}$ is significantly perturbed at $E>0.65 \mathrm{~V}$ by the appearance of small gold islands which are created by the lifting of the reconstruction of $\mathrm{Au}(111)$ (Figure 6f-h).

The above in situ dynamic process, in combination with results from cyclic voltammetry, showed that the phase transition of chiral $p$-CPAEt adlayers on the gold electrode were purely driven by changes on the electrode potential and not by redox-modifications of the adsorbed species. The formation of self-assembled chiral adlayers on a surface is the result of a delicate interplay between adsorbate-adsorbate and adsorbate-substrate interactions, which involve $d-\pi^{*}$ interaction, hydrogen bonding, and van der Waals forces, etc. A change of either one or both will be the reason for a structural transition. ${ }^{33}$ The stripe pattern in $0.65 \mathrm{~V}<E<0.8 \mathrm{~V}$ followed the main symmetry direction $\langle 110\rangle$ of the underlying hexagonal $\mathrm{Au}(111)$ lattice directly. In contrast, the network patterns in $0.2 \mathrm{~V}<E \leq 0.65 \mathrm{~V}$ arranged along the $\sqrt{ } 7$ direction tilted by the opposite sign of $\sim 11^{\circ}$ with the main symmetry direction $\langle 121\rangle$ of the surface. These mean that the $d-\pi^{*}$ interaction between the conjugated systems of $p$-CPAEt and Au substrate takes the important role for the stripe pattern in the positively charged potential region, but the hydrogen bond and van der Waals forces between the adsorbed $p$-CPAEt molecules are more important for the network patterns in the relatively negatively charged potential. Such a sensitive equilibrium change between adsorbate-adsorbate and adsorbate-substrate interactions may result in the phase transistion. From the stripe pattern to the network pattern, a reduced electrostatic interaction between $p$-CPAEt and the substrate underneath holds somewhat less $p$-CPAEt at the surface, i.e., the coverage decreases. Similar phenomena were reported for porphyrine on $\mathrm{Cu}(111)^{33}$ and for 4-methylbenzenethiol on $\mathrm{Au}(111){ }^{34}$

\section{CONCLUSION}

In situ ECSTM measurements have been performed for $p$-CPAEt molecules on $\mathrm{Au}(111)$ electrode in $0.1 \mathrm{M} \mathrm{HClO}_{4}$ in order to develop a detailed molecular-level understanding of steadystate chiral supramolecular nanostructures and their phase transition tuned by applied substrate potential. It was demonstrated that distinctly different chiral adlayers could be created by applied electrode potential, and studied by an in situ STM technique. The different ordered phases were the result of a delicate interplay between adsorbate-adsorbate and adsorbate - substrate interactions, which involve $\mathrm{d}-\pi^{*}$ interaction, hydrogen bonding, and van der Waals forces.

At $0.65 \mathrm{~V}<E<0.8 \mathrm{~V}$, the closely packed chiral stripe patterns have been found on a $\mathrm{Au}(111)$ electrode. At a more negatively charged electrode surface with the potential range $0.2 \mathrm{~V}<E \leq$ $0.65 \mathrm{~V}$, two types of chiral network I and II were observed which were typically favored on reconstructed and unreconstructed surfaces, respectively. On the basis of the adlayer structures and chirality, the adsorption models were tentatively suggested for each type of chiral nanostructure. The 2D ordered behaviors of these chiral adlayers are mainly governed by adsorption-induced chiral $p$-CPAET species and the lateral hydrogen-bonding interaction $(\mathrm{C}-\mathrm{H} \cdots \mathrm{N} \equiv \mathrm{C})$. The mirror positions of $-\mathrm{C} \equiv \mathrm{N}$ would lead to a switch in supramolecular assembly directions.

On the other hand, the dynamic processes of the phase transition of chiral supramolecular nanostructures have been investigated. Time-dependent sequences show that the stripe patterns could be transformed into network structures within the thermodynamically stable potential region of the latter, but the transforming process would be delayed by the reconstruction lines. Furthermore, if the electrode potential is scanned positively until the region of the thermodynamic stability of the stripe 
pattern, the network nanostructure dissolved and the stripe pattern reappeared again. These dynamic processes, in combination with electrochemical cyclic voltammetry, showed that the transformation from the chiral stripe nanostructure to the chiral network structure was purely potential-dependent and reversible. It means that the chiral supramolecular nanostructure could be controlled by tuning the electrode potential in the electrochemical environment.

The successful creation of chiral adlayers and in situ monitoring of transition process driven by the electrochemical potential represent the deliberate control of molecules on substrates. The work not only demonstrated the possibility and ability of tuning molecular chiral structures at the nanoscale level by external stimuli, the applied electrode potential, but also provided useful information for closely related fields, such as bottom-up assembly of molecule-scale building blocks on surfaces, electrocatalysis, chiral synthesis, and discrimination, etc.

\section{ACKNOWLEDGMENT}

This work was supported by the National Natural Science Foundation of China (20807049), the National Basic Research Program of China (Grant 2009CB421606), and Research Center Jülich. G.-j.S. acknowledges support from Alexander von Humboldt Foundation. R.A-S. acknowledges support from the German Academic Exchange Agency.

\section{SUPPORTING INFORMATION AVAILABLE}

Additional information as noted in the text. This material is available free of charge via the Internet at http://pubs.acs.org.

Received for review April 19, 2009. Accepted October 1, 2009.

AC901530G 